\title{
Two ways for solving a class of rational second-order difference equations
}

Stevo Stević $\mathfrak{c}^{1,2,3^{*}}$

"Correspondence: sstevic@ptt.rs 1 Mathematical Institute of the Serbian Academy of Sciences, Beograd, Serbia

${ }^{2}$ Department of Medical Research, China Medical University Hospital, China Medical University, Taichung, Taiwan, Republic of China

Full list of author information is available at the end of the article

\begin{abstract}
We consider a class of second-order rational difference equations with two parameters, and we show, in two ways, that the class of equations is solvable in closed form. One way is based on using an invariant for the class of equations, whereas the second one is based on the method of substitution. The main results extend and complement some previous ones in the literature.
\end{abstract}

MSC: Primary 39A20; secondary 39A06; 39A45

Keywords: Difference equation; Solvable equation; Invariant; Method of substitution

\section{Introduction}

We use the following standard notations $\mathbb{N}, \mathbb{N}_{0}, \mathbb{Z}, \mathbb{R}, \mathbb{C}$, for natural, nonnegative, whole, real and complex numbers, respectively.

Finding closed-form formulas for solutions to difference equations started at the beginning of the eighteenth century by de Moivre, who knew to solve homogeneous linear difference equations with constant coefficients $([1,2])$. He first solved the difference equation

$$
x_{n+2}=\widetilde{a} x_{n+1}+\widetilde{b} x_{n}, \quad n \in \mathbb{N}_{0},
$$

by showing that under the conditions $\widetilde{b} \neq 0$ and $\widetilde{a}^{2} \neq-4 \widetilde{b}$, its general solution is given by the following formula:

$$
x_{n}=\frac{\left(x_{1}-\lambda_{2} x_{0}\right) \lambda_{1}^{n}+\left(\lambda_{1} x_{0}-x_{1}\right) \lambda_{2}^{n}}{\lambda_{1}-\lambda_{2}}, \quad n \in \mathbb{N}_{0}
$$

where $\lambda_{1}$ and $\lambda_{2}$ are roots of the polynomial $P_{2}(\lambda)=\lambda^{2}-\tilde{a} \lambda-\widetilde{b}$. Equation (2) is called the de Moivre formula, whereas the polynomial $P_{2}$ is called the characteristic polynomial associated to the linear equation (1).

Methods and ideas by de Moivre were later developed by Euler ([3]). The study was continued by Lagrange (see, e.g., $[4,5]$ ), Laplace (see, e.g., [6]), and many other scientists. Several methods for solving some classes of nonlinear difference equations in closed form had been known to Laplace yet ([6]).

(c) The Author(s) 2019. This article is distributed under the terms of the Creative Commons Attribution 4.0 International License (http://creativecommons.org/licenses/by/4.0/), which permits unrestricted use, distribution, and reproduction in any medium, provided you give appropriate credit to the original author(s) and the source, provide a link to the Creative Commons license, and indicate if changes were made. 
From [6] we also see that Laplace essentially knew to solve the class of bilinear difference equations, that is, the following one:

$$
x_{n+1}=\frac{\alpha x_{n}+\beta}{\gamma x_{n}+\delta}, \quad n \in \mathbb{N}_{0}
$$

where $\alpha, \beta, \gamma, \delta$ are given numbers. Let us mention that it is usually assumed that $\alpha \delta-\beta \gamma \neq 0 \neq \gamma$ to avoid dealing with the trivial and linear equations obtained from Eq. (3) (the equation with $\gamma=0$ was solved by Lagrange in [4]; moreover, the first-order linear difference equation with nonconstant coefficients was also solved therein). For more historical details on some classical difference equations including bilinear one, see [7]. Hence, Eq. (3) is regarded as one of the basic solvable nonlinear difference equations, and can be found in many books ([8-14]; see also [15]). The equation frequently appears in papers on the solvability of difference equations (see, e.g., the recent papers [7, 16-19]).

Note that, by using the change of variables

$$
\tilde{x}_{n}=\gamma x_{n}+\delta, \quad n \in \mathbb{N}_{0}
$$

Equation (3) is transformed to the following one

$$
\widetilde{x}_{n+1}=\widetilde{\alpha}+\frac{\widetilde{\beta}}{\widetilde{x}_{n}}, \quad n \in \mathbb{N}_{0}
$$

By using the following change of variables

$$
\widetilde{x}_{n}=\frac{u_{n}}{u_{n-1}}, \quad n \in \mathbb{N}_{0},
$$

from Eq. (4) we get

$$
u_{n+1}=\widetilde{\alpha} u_{n}+\widetilde{\beta} u_{n-1}, \quad n \in \mathbb{N}_{0},
$$

which is an equation of the form in (1), so, solvable one, implying the solvability of Eq. (3) (see, e.g., also $[13,15,16])$.

Nowadays, a part of the scientific community seems uses only computers and symbolic algebra for getting some results on solvability of various equations, including difference ones. This causes several problems, including some on originality of such obtained results. In some of our papers, among other things, we have discussed such problems (see, e.g., $[7,16-22])$. Many of the known solvable difference equations and systems can be transformed to linear ones, which are solvable (for solvability of linear difference equations see, e.g., $[8,9,14])$. This motivated some authors to apply and develop the method of transformation/substitution (see, e.g., [20-29]). Many applications of solvable difference equations, can be found in the literature which shows their importance (see, e.g., [2, 5, 6, 9-14, 30-36]). For some related results see also [37-42].

Now we give a formal definition of solvability of a difference equation. 
Definition 1 We say that a difference equation is solvable in closed form if there is a finite number of closed-form formulas from which any well-defined solution to the equation can be obtained.

Some closed-form formulas for solutions to the difference equation

$$
x_{n+1}=x_{n}+\frac{x_{n}}{x_{n}-x_{n-1}}, \quad n \in \mathbb{N}_{0},
$$

appeared in [43], where they were proved by induction. However, there was said nothing about the way for obtaining the formulas, which motivated us to find theoretical explanations for getting them. Some explanations, including a method for getting the formulas, have been given, recently, in [44]. This was done in a quite unexpected way. Namely, by using an invariant for Eq. (5). The following solvability result holds $([43,44])$.

Theorem 1 Consider the difference equation

$$
x_{n+1}=x_{n}+\frac{d x_{n}}{x_{n}-x_{n-1}}, \quad n \in \mathbb{N}_{0},
$$

where $d \in \mathbb{C} \backslash\{0\}$, and $x_{-1}, x_{0} \in \mathbb{C}$. Then the general solution to Eq. (6) is given by the following formula:

$$
\begin{aligned}
x_{n}= & \left(x_{0}-x_{-1}+\frac{d x_{0}}{x_{0}-x_{-1}}\right) \frac{n}{2}+\frac{1}{8}\left(6 x_{0}+2 x_{-1}+\frac{2 d x_{0}}{x_{0}-x_{-1}}-d\right) \\
& +\left(2 x_{0}-2 x_{-1}-\frac{2 d x_{0}}{x_{0}-x_{-1}}+d\right) \frac{(-1)^{n}}{8}+\frac{d}{4} n^{2},
\end{aligned}
$$

for $n \geq-1$.

Invariants for difference equations can be useful in their investigations. But it is usually difficult to find those which can be of any use. Invariants for several classes of difference equations and systems of difference equations can be found, e.g., in [45-50] (see also the related references therein). They can be useful in finding general solutions to some classes of difference equations, although it is not quite a common situation. Some examples of difference equations which are solved by using invariants can be found in $[44,51]$ and [52].

Now we define notion of being an invariant for a difference equation.

Definition 2 If for every solution $\left(x_{n}\right)_{n \geq-k}$ to the difference equation

$$
x_{n+s}=f\left(x_{n+s-1}, x_{n+s-2}, \ldots, x_{n}\right), \quad n \geq-k,
$$

where $s, k \in \mathbb{N}$, there is a function $I: \mathbb{R}^{l}\left(\right.$ or $\left.\mathbb{C}^{l}\right) \rightarrow \mathbb{R}($ or $\mathbb{C}$ ) and a constant $c$, such that

$$
I\left(x_{n}, x_{n+1}, \ldots, x_{n+l-1}\right)=c, \quad \text { for } n \geq-k
$$

then we say that $I$ is an invariant for Eq. (8). 
Motivated by [44] here we study an extension of Eq. (6). Namely, we show that the following generalization of Eq. (6)

$$
x_{n+1}=a x_{n}+\frac{d x_{n}}{x_{n}-a x_{n-1}}, \quad n \in \mathbb{N}_{0},
$$

where parameters $a$ and $d$, and initial values $x_{-1}$ and $x_{0}$ are given numbers, can also be solved in closed form, extending and complementing the main result in [44].

We prove this by using two methods. First, this is proved by using an invariant for the difference equation. Since in this way is obtained a closed-form formula for general solution to the equation, this suggests that it might be some other, more standard, methods for getting the formula. Bearing in mind some of our previous investigations on solvability of difference equations and systems of difference equations (see, e.g., [20-22, 24-29] and number of related references therein), the first natural choice is the method of substitution. Employing some suitably chosen changes of variables we also show the solvability of Eq. (9), confirming the guess.

\section{Main results}

Our main results are stated and proved in this section. As we have already mentioned, here we present two methods for solving difference equation (9). The first one essentially uses an invariant for the equation together with a nice trick, while the second one is based on the method of substitution where a chain of substitutions is used to get general solution to the equation.

First note that if $d=0$, then Eq. (9) becomes

$$
x_{n+1}=a x_{n}, \quad n \in \mathbb{N}_{0},
$$

from which it easily follows that

$$
x_{n}=a^{n} x_{0}, \quad n \in \mathbb{N}_{0} .
$$

Hence, from now on we will assume that $d \neq 0$.

Also note that if $a=0$, then Eq. (9) becomes

$$
x_{n+1}=x_{n} \frac{d}{x_{n}}, \quad n \in \mathbb{N}_{0}
$$

from which it easily follows that every solution to the equation is well-defined if and only if $x_{0} \neq 0$, and if $x_{0} \neq 0$, then $x_{n}=d, n \in \mathbb{N}$. Hence, from now on we will also assume that $a \neq 0$.

Bearing in mind Definition 1, we see also that solutions which are not well-defined should not be taken into the consideration. This means that, for every well-defined solution $\left(x_{n}\right)_{n \geq-1}$ to the Eq. (9),

$$
x_{n} \neq a x_{n-1}, \quad n \in \mathbb{N}_{0} .
$$




\subsection{Solving Eq. (9) by using an invariant}

Here we use our idea in [44]. First, note that, from (9), we have

$$
\left(x_{n+1}-a x_{n}\right)\left(x_{n}-a x_{n-1}\right)-d x_{n}=0, \quad n \in \mathbb{N}_{0} .
$$

Let

$$
I(u, v, w)=(w-a v)(v-a u)-d v .
$$

Then from (11) we see that function (12) is an invariant for Eq. (9).

Now note that

$$
I\left(x_{n-1}, x_{n}, x_{n+1}\right)=a I\left(x_{n-2}, x_{n-1}, x_{n}\right), \quad n \in \mathbb{N},
$$

that is,

$$
\left(x_{n+1}-a x_{n}\right)\left(x_{n}-a x_{n-1}\right)-d x_{n}=a\left(x_{n}-a x_{n-1}\right)\left(x_{n-1}-a x_{n-2}\right)-a d x_{n-1} \text {, }
$$

for $n \in \mathbb{N}$, from which it follows that

$$
\left(x_{n}-a x_{n-1}\right)\left(x_{n+1}-a x_{n}-a x_{n-1}+a^{2} x_{n-2}-d\right), \quad n \in \mathbb{N} \text {. }
$$

Since (10) must hold, from (13), we obtain

$$
x_{n+1}-a x_{n}-a x_{n-1}+a^{2} x_{n-2}=d, \quad n \in \mathbb{N} \text {. }
$$

The zeros of the characteristic polynomial

$$
p_{3}(\lambda)=\lambda^{3}-a \lambda^{2}-a \lambda+a^{2}=(\lambda-a)\left(\lambda^{2}-a\right),
$$

associated with (14), are

$$
\lambda_{1}=a, \quad \lambda_{2}=\sqrt{a}, \quad \lambda_{3}=-\sqrt{a} .
$$

Case $a=1$ was considered in [44]. Hence, the case is omitted here, and we assume that $a \in \mathbb{C} \backslash\{1,0\}$. In this case the characteristic roots in (16) are mutually different. Hence, the general solution to the homogeneous equation

$$
x_{n+1}-a x_{n}-a x_{n-1}+a^{2} x_{n-2}=0
$$

is

$$
x_{n}^{h}=c_{1} a^{n}+c_{2}(\sqrt{a})^{n}+c_{3}(-\sqrt{a})^{n}, \quad n \geq-1,
$$

whereas a solution to Eq. (14) can be found in the form

$$
x_{n}^{p}=\widetilde{c}, \quad n \geq-1,
$$


from which it is easily found that

$$
x_{n}^{p}=\frac{d}{(1-a)^{2}}, \quad n \geq-1 .
$$

Hence,

$$
x_{n}=c_{1} a^{n}+c_{2}(\sqrt{a})^{n}+c_{3}(-\sqrt{a})^{n}+\frac{d}{(1-a)^{2}}, \quad n \geq-1 .
$$

Using (17) for $n=-1,0,1$, respectively, we get

$$
\begin{aligned}
& a^{-1} c_{1}+(\sqrt{a})^{-1} c_{2}-(\sqrt{a})^{-1} c_{3}=x_{-1}-\frac{d}{(1-a)^{2}}, \\
& c_{1}+c_{2}+c_{3}=x_{0}-\frac{d}{(1-a)^{2}}, \\
& a c_{1}+\sqrt{a} c_{2}-\sqrt{a} c_{3}=x_{1}-\frac{d}{(1-a)^{2}} .
\end{aligned}
$$

The determinant of system (18) is

$$
\Delta=\left|\begin{array}{ccc}
a^{-1} & (\sqrt{a})^{-1} & -(\sqrt{a})^{-1} \\
1 & 1 & 1 \\
a & \sqrt{a} & -\sqrt{a}
\end{array}\right|=\frac{2(a-1)}{\sqrt{a}} .
$$

Let

$$
\tilde{x}_{j}=x_{j}-\frac{d}{(1-a)^{2}}, \quad j=-1,0,1 \text {. }
$$

By a well-known theorem, using (19) and (20), and after some calculations, we have

$$
\begin{aligned}
c_{1} & =\frac{1}{\Delta}\left|\begin{array}{ccc}
\tilde{x}_{-1} & (\sqrt{a})^{-1} & -(\sqrt{a})^{-1} \\
\tilde{x}_{0} & 1 & 1 \\
\tilde{x}_{1} & \sqrt{a} & -\sqrt{a}
\end{array}\right|=\frac{-2 \sqrt{a \tilde{x}_{-1}+\frac{2}{\sqrt{a}} \tilde{x}_{1}}}{\Delta} \\
& =\frac{a\left(x_{0}-x_{-1}\right)+\frac{d x_{0}}{x_{0}-a x_{-1}}+\frac{d}{a-1}}{a-1}, \\
c_{2} & =\frac{1}{\Delta}\left|\begin{array}{ccc}
a^{-1} & \tilde{x}_{-1} & -(\sqrt{a})^{-1} \\
1 & \tilde{x}_{0} & 1 \\
a & \tilde{x}_{1} & -\sqrt{a}
\end{array}\right|=\frac{(\sqrt{a}+a) \tilde{x}_{-1}+\left(\sqrt{a}-\frac{1}{\sqrt{a}}\right) \tilde{x}_{0}-\left(\frac{1}{a}+\frac{1}{\sqrt{a}}\right) \tilde{x}_{1}}{\Delta} \\
& =\frac{(1+\sqrt{a})\left(a x_{-1}-x_{0}\right)-\frac{1+\sqrt{a}}{\sqrt{a}} \frac{d x_{0}}{x_{0}-a x_{-1}}-\frac{d(\sqrt{a}+1)^{2}}{\sqrt{a}(a-1)}}{2(a-1)},
\end{aligned}
$$


and

$$
\begin{aligned}
c_{3} & =\frac{1}{\Delta}\left|\begin{array}{ccc}
a^{-1} & (\sqrt{a})^{-1} & \tilde{x}_{-1} \\
1 & 1 & \tilde{x}_{0} \\
a & \sqrt{a} & \tilde{x}_{1}
\end{array}\right|=\frac{(\sqrt{a}-a) \tilde{x}_{-1}+\left(\sqrt{a}-\frac{1}{\sqrt{a}}\right) \tilde{x}_{0}+\left(\frac{1}{a}-\frac{1}{\sqrt{a}}\right) \tilde{x}_{1}}{\Delta} \\
& =\frac{(\sqrt{a}-1)\left(x_{0}-a x_{-1}\right)+\frac{1-\sqrt{a}}{\sqrt{a}} \frac{d x_{0}}{x_{0}-a x_{-1}}+\frac{d(\sqrt{a}-1)^{2}}{\sqrt{a}(a-1)}}{2(a-1)} .
\end{aligned}
$$

Using (21)-(23) in (17), we get

$$
\begin{aligned}
x_{n}= & \left(\frac{\left.a\left(x_{0}-x_{-1}\right)+\frac{d x_{0}}{x_{0}-a x_{-1}}+\frac{d}{a-1}\right) a^{n}}{a-1}\right)(\sqrt{a})^{n} \\
& +\left(\frac{(1+\sqrt{a})\left(a x_{-1}-x_{0}\right)-\frac{1+\sqrt{a}}{\sqrt{a}} \frac{d x_{0}}{x_{0}-a x_{-1}}-\frac{d(\sqrt{a}+1)^{2}}{\sqrt{a}(a-1)}}{2(a-1)}\right)(-\sqrt{a})^{n}+\frac{d}{(a-1)^{2}}, \\
& +\left(\frac{(\sqrt{a}-1)\left(x_{0}-a x_{-1}\right)+\frac{1-\sqrt{a}}{\sqrt{a}} \frac{d x_{0}}{x_{0}-a x_{-1}}+\frac{d(\sqrt{a}-1)^{2}}{\sqrt{a}(a-1)}}{2(a-1)}\right)
\end{aligned}
$$

for $n \geq-1$.

Now we check that the sequences given by (24) satisfy Eq. (9). Let

$$
\begin{aligned}
\widehat{x}_{n}:= & \left(\frac{a\left(x_{0}-x_{-1}\right)+\frac{d x_{0}}{x_{0}-a x_{-1}}+\frac{d}{a-1}}{a-1}\right) a^{n} \\
& +\left(\frac{(1+\sqrt{a})\left(a x_{-1}-x_{0}\right)-\frac{1+\sqrt{a}}{\sqrt{a}} \frac{d x_{0}}{x_{0}-a x_{-1}}-\frac{d(\sqrt{a}+1)^{2}}{\sqrt{a}(a-1)}}{2(a-1)}\right)(\sqrt{a})^{n} \\
& +\left(\frac{(\sqrt{a}-1)\left(x_{0}-a x_{-1}\right)+\frac{1-\sqrt{a}}{\sqrt{a}} \frac{d x_{0}}{x_{0}-a x_{-1}}+\frac{d(\sqrt{a}-1)^{2}}{\sqrt{a}(a-1)}}{2(a-1)}\right)(-\sqrt{a})^{n}+\frac{d}{(a-1)^{2}},
\end{aligned}
$$

for $n \geq-1$.

By the way of getting (24) it is clear that

$$
\widehat{x}_{-1}=x_{-1} \text { and } \widehat{x}_{0}=x_{0} \text {. }
$$

From (25) we have

$$
\begin{aligned}
\widehat{x}_{2 n-1}:= & \left(\frac{a\left(x_{0}-x_{-1}\right)+\frac{d x_{0}}{x_{0}-a x_{-1}}+\frac{d}{a-1}}{a-1}\right) a^{2 n-1} \\
& +\left(\frac{(1+\sqrt{a})\left(a x_{-1}-x_{0}\right)-\frac{1+\sqrt{a}}{\sqrt{a}} \frac{d x_{0}}{x_{0}-a x_{-1}}-\frac{d(\sqrt{a}+1)^{2}}{\sqrt{a}(a-1)}}{2(a-1)}\right)(\sqrt{a})^{2 n-1} \\
& -\left(\frac{(\sqrt{a}-1)\left(x_{0}-a x_{-1}\right)+\frac{1-\sqrt{a}}{\sqrt{a}} \frac{d x_{0}}{x_{0}-a x_{-1}}+\frac{d(\sqrt{a}-1)^{2}}{\sqrt{a}(a-1)}}{2(a-1)}\right)(\sqrt{a})^{2 n-1}+\frac{d}{(a-1)^{2}} \\
= & \left(\frac{a\left(x_{0}-x_{-1}\right)+\frac{d x_{0}}{x_{0}-a x_{-1}}+\frac{d}{a-1}}{a-1}\right) a^{2 n-1} \\
& -\left(a x_{0}-a^{2} x_{-1}+\frac{d x_{0}}{x_{0}-a x_{-1}}+\frac{d(a+1)}{a-1}\right) \frac{a^{n-1}}{a-1}+\frac{d}{(a-1)^{2}}
\end{aligned}
$$


and

$$
\begin{aligned}
\widehat{x}_{2 n}:= & \left(\frac{\left.a\left(x_{0}-x_{-1}\right)+\frac{d x_{0}}{x_{0}-a x_{-1}}+\frac{d}{a-1}\right) a^{2 n}}{a-1}\right) a^{n} \\
& +\left(\frac{(1+\sqrt{a})\left(a x_{-1}-x_{0}\right)-\frac{1+\sqrt{a}}{\sqrt{a}} \frac{d x_{0}}{x_{0}-a x_{-1}}-\frac{d(\sqrt{a}+1)^{2}}{\sqrt{a}(a-1)}}{2(a-1)}\right) a^{n}+\frac{d}{(a-1)^{2}} \\
& +\left(\frac{(\sqrt{a}-1)\left(x_{0}-a x_{-1}\right)+\frac{1-\sqrt{a}}{\sqrt{a}} \frac{d x_{0}}{x_{0}-a x_{-1}}+\frac{d(\sqrt{a}-1)^{2}}{\sqrt{a}(a-1)}}{2(a-1)}\right) a^{2 n} \\
= & \left(\frac{a\left(x_{0}-x_{-1}\right)+\frac{d x_{0}}{x_{0}-a x_{-1}}+\frac{d}{a-1}}{a-1}+\frac{2 d}{a-1}\right) \frac{a^{n}}{a-1}+\frac{d}{(a-1)^{2}} .
\end{aligned}
$$

We also have

$$
\left.\widehat{x}_{2 n}=\left(\frac{\left(x_{0}-x_{-1}\right) a^{n+1}-\left(x_{0}-a x_{-1}\right)}{\left(x_{0}-a x_{-1}\right)(a-1)^{2}}\right)\left((a-1) x_{0}+a(1-a) x_{-1}+d\right) a^{n}-d\right),
$$

for $n \geq-1$.

From (27) and (28), we have

$$
\widehat{x}_{2 n}-a \widehat{x}_{2 n-1}=\frac{\left((a-1) x_{0}+a(1-a) x_{-1}+d\right) a^{n}-d}{a-1},
$$

from which it follows that

$$
\begin{aligned}
a+\frac{d}{\widehat{x}_{2 n}-a \widehat{x}_{2 n-1}} & =a+\frac{d(a-1)}{\left((a-1) x_{0}+a(1-a) x_{-1}+d\right) a^{n}-d} \\
& =\frac{\left((a-1) x_{0}+a(1-a) x_{-1}+d\right) a^{n+1}-d}{\left((a-1) x_{0}+a(1-a) x_{-1}+d\right) a^{n}-d} .
\end{aligned}
$$

By using (29) and (30), we have

$$
\begin{aligned}
\widehat{x}_{2 n} & \left(a+\frac{d}{\widehat{x}_{2 n}-a \widehat{x}_{2 n-1}}\right) \\
= & \left.\left(\frac{\left(x_{0}-x_{-1}\right) a^{n+1}-\left(x_{0}-a x_{-1}\right)}{\left(x_{0}-a x_{-1}\right)(a-1)^{2}}\right)\left((a-1) x_{0}+a(1-a) x_{-1}+d\right) a^{n+1}-d\right) \\
= & \left(\frac{a\left(x_{0}-x_{-1}\right)+\frac{d x_{0}}{x_{0}-a x_{-1}}+\frac{d}{a-1}}{a-1}\right) a^{2 n+1} \\
& -\left(a x_{0}-a^{2} x_{-1}+\frac{d x_{0}}{x_{0}-a x_{-1}}+\frac{d(a+1)}{a-1}\right) \frac{a^{n}}{a-1}+\frac{d}{(a-1)^{2}} \\
= & \widehat{x}_{2 n+1} .
\end{aligned}
$$

From (28) and (32), we have

$$
\widehat{x}_{2 n+1}-a \widehat{x}_{2 n}=\frac{d\left(\left(x_{0}-x_{-1}\right) a^{n+1}-\left(x_{0}-a x_{-1}\right)\right)}{\left(x_{0}-a x_{-1}\right)(a-1)},
$$


from which it follows that

$$
\begin{aligned}
a+\frac{d}{\widehat{x}_{2 n+1}-a \widehat{x}_{2 n}} & =a+\frac{\left(x_{0}-a x_{-1}\right)(a-1)}{\left(x_{0}-x_{-1}\right) a^{n+1}-\left(x_{0}-a x_{-1}\right)} \\
& =\frac{\left(x_{0}-x_{-1}\right) a^{n+2}-\left(x_{0}-a x_{-1}\right)}{\left(x_{0}-x_{-1}\right) a^{n+1}-\left(x_{0}-a x_{-1}\right)} .
\end{aligned}
$$

Using (31) and (34), we have

$$
\begin{aligned}
\widehat{x}_{2 n+1} & \left(a+\frac{d}{\widehat{x}_{2 n+1}-a \widehat{x}_{2 n}}\right) \\
= & \left(\frac{\left((a-1)\left(x_{0}-a x_{-1}\right)+d\right) a^{n+1}-d}{(a-1)^{2}\left(x_{0}-a x_{-1}\right)}\right)\left(\left(x_{0}-x_{-1}\right) a^{n+2}-\left(x_{0}-a x_{-1}\right)\right) \\
= & \left(\frac{a\left(x_{0}-x_{-1}\right)+\frac{d x_{0}}{x_{0}-a x_{-1}}+\frac{d}{a-1}}{a-1}\right) a^{2 n+2} \\
& -\left(x_{0}-a x_{-1}+\frac{d x_{0}}{x_{0}-a x_{-1}}+\frac{2 d}{a-1}\right) \frac{a^{n+1}}{a-1}+\frac{d}{(a-1)^{2}} \\
= & \widehat{x}_{2 n+2} .
\end{aligned}
$$

Equations (26), (33), (35) together with induction show that (25) is a solution to (9). Hence, we have the following theorem, which complements Theorem 1.

Theorem 2 Consider Eq. (9). If $a \neq 1$, then the general solution to the equation is given by Eq. (24).

\subsection{Solving Eq. (9) by the method of substitution}

Before we start describing the procedure for finding general solution to Eq. (9), which is based on the method of substitution (use of some changes of variables), we would like to point out that if

$$
x_{n_{0}}=0 \quad \text { and } \quad x_{n_{0}} \neq a x_{n_{0}-1},
$$

for some $n_{0} \in \mathbb{N}_{0}$ (of course, we regard that terms $x_{n}$ are defined for $-1 \leq n \leq n_{0}$ ), then from (9) it follows that $x_{n_{0}+1}=0$, from which along with (36) it follows that $x_{n_{0}+1}-a x_{n_{0}}=0$, implying that $x_{n_{0}+2}$ is not defined. Hence, such solutions are not well-defined. So, from now on we may also assume that

$$
x_{n} \neq 0,
$$

for every $n \in \mathbb{N}_{0}$. Value of $x_{-1}$ can be equal to zero and it essentially does not effect the final solution. However, since it effects some steps of this procedure for finding general solution, we will also assume that $x_{-1} \neq 0$.

Our first idea is to write Eq. (9) in the form of a product-type difference equations, which along with systems of product-type difference equations have been considerably studied (see, e.g., $[28,53-55]$ and the related references therein). 
So, first note that from (9) we have

$$
x_{n+1}-a x_{n}=\frac{d x_{n}}{x_{n}-a x_{n-1}}, \quad n \in \mathbb{N}_{0} .
$$

Hence

$$
x_{n}-a x_{n-1}=\frac{d x_{n-1}}{x_{n-1}-a x_{n-2}}, \quad n \in \mathbb{N} \text {. }
$$

By using (39) in (38), it follows that

$$
x_{n+1}-a x_{n}=\frac{x_{n}}{x_{n-1}}\left(x_{n-1}-a x_{n-2}\right), \quad n \in \mathbb{N} .
$$

Equation (40) can be written in the form

$$
\frac{x_{n+1}}{x_{n}}=a+1-a \frac{x_{n-2}}{x_{n-1}}, \quad n \in \mathbb{N} .
$$

By using the change of variables

$$
y_{n}=\frac{x_{n}}{x_{n-1}}, \quad n \in \mathbb{N}_{0},
$$

Equation (41) becomes

$$
y_{n+1}=a+1-\frac{a}{y_{n-1}}, \quad n \in \mathbb{N} .
$$

Now note that the difference equation (43) is an equation with interlacing indices of order two $([17,20])$. This means that the sequences

$$
y_{m}^{(i)}:=y_{2 m+i}
$$

for $m \in \mathbb{N}_{0}$ and $i=0,1$, are two solutions to the first-order difference equation

$$
z_{m+1}=a+1-\frac{a}{z_{m}}, \quad m \in \mathbb{N}_{0}
$$

Equation (45) is a special case of the bilinear difference equation, or more precisely, of Eq. (4). Hence, it is solvable, implying solvability of Eq. (43), which along with the change of variables (42) implies solvability of Eq. (40).

There are two cases to be considered: (1) $a=1$; (2) $a \neq 1$.

Case $a=1$. In this case Eq. (45) becomes

$$
z_{m+1}=2-\frac{1}{z_{m}}, \quad m \in \mathbb{N}_{0}
$$

By using the change of variables

$$
z_{m}=\frac{u_{m}}{u_{m-1}}, \quad m \in \mathbb{N}_{0},
$$


where we assume that $u_{-1}$ is a nonzero number, we get

$$
u_{m+1}=2 u_{m}-u_{m-1}, \quad m \in \mathbb{N}_{0} .
$$

Since the roots of the characteristic polynomial $\widetilde{P}_{2}(\lambda)=\lambda^{2}-2 \lambda+1$ associated with Eq. (48) are $\lambda_{1}=\lambda_{2}=1$, then the general solution to Eq. (48) is

$$
u_{m}=\widehat{c}_{1} m+\widehat{c}_{2}, \quad m \geq-1
$$

from which it follows that

$$
u_{m}=\left(u_{0}-u_{-1}\right) m+u_{0}=u_{0}(m+1)-u_{-1} m, \quad m \geq-1 .
$$

By using (50) in (47), we obtain

$$
z_{m}=\frac{u_{0}(m+1)-u_{-1} m}{u_{0} m-u_{-1}(m-1)}=\frac{z_{0}(m+1)-m}{z_{0} m-m+1}, \quad m \in \mathbb{N}_{0}
$$

From (44) and (51), it follows that

$$
y_{2 m+i}=y_{m}^{(i)}=\frac{y_{0}^{(i)}(m+1)-m}{y_{0}^{(i)} m-m+1}=\frac{y_{i}(m+1)-m}{y_{i} m-m+1},
$$

for $m \in \mathbb{N}_{0}$ and $i=0,1$, that is,

$$
\begin{aligned}
y_{2 m}= & \frac{y_{0}(m+1)-m}{y_{0} m-m+1}=\frac{x_{0}(m+1)-x_{-1} m}{x_{0} m-x_{-1}(m-1)}, \\
y_{2 m+1}= & \frac{y_{1}(m+1)-m}{y_{1} m-m+1}=\frac{x_{1}(m+1)-x_{0} m}{x_{1} m-x_{0}(m-1)}=\frac{\left(x_{0}+\frac{d x_{0}}{x_{0}-x_{-1}}\right)(m+1)-x_{0} m}{\left(x_{0}+\frac{d x_{0}}{x_{0}-x_{-1}}\right) m-x_{0}(m-1)} \\
& =\frac{d(m+1)+x_{0}-x_{-1}}{d m+x_{0}-x_{-1}}, \quad m \in \mathbb{N}_{0} .
\end{aligned}
$$

From (42) we have

$$
x_{n}=x_{-1} \prod_{j=0}^{n} y_{j}, \quad n \geq-1
$$

Hence, (52)-(54) yield

$$
\begin{aligned}
x_{2 n-1} & =x_{-1} \prod_{j=0}^{2 n-1} y_{j}=x_{-1} \prod_{i=0}^{n-1} y_{2 i} \prod_{i=0}^{n-1} y_{2 i+1} \\
& =x_{-1} \prod_{i=0}^{n-1} \frac{x_{0}(i+1)-x_{-1} i}{x_{0} i-x_{-1}(i-1)} \prod_{i=0}^{n-1} \frac{d(i+1)+x_{0}-x_{-1}}{d i+x_{0}-x_{-1}} \\
& =x_{-1} \frac{x_{0} n-x_{-1}(n-1)}{x_{-1}} \cdot \frac{d n+x_{0}-x_{-1}}{x_{0}-x_{-1}} \\
& =\left(\left(x_{0}-x_{-1}\right) n+x_{-1}\right)\left(\frac{d n}{x_{0}-x_{-1}}+1\right)
\end{aligned}
$$




$$
=x_{-1}+\left(x_{0}-x_{-1}+\frac{d x_{-1}}{x_{0}-x_{-1}}\right) n+d n^{2}
$$

for $n \in \mathbb{N}_{0}$.

Employing (42), (52), (54) and (55), we get

$$
\begin{aligned}
x_{2 n} & =x_{-1} \prod_{j=0}^{2 n} y_{j}=x_{2 n-1} y_{2 n} \\
& =\left(\left(x_{0}-x_{-1}\right) n+x_{-1}\right)\left(\frac{d n}{x_{0}-x_{-1}}+1\right) \frac{x_{0}(n+1)-x_{-1} n}{x_{0} n-x_{-1}(n-1)} \\
& =\left(\frac{d n}{x_{0}-x_{-1}}+1\right)\left(\left(x_{0}-x_{-1}\right) n+x_{0}\right) \\
& =x_{0}+\left(x_{0}-x_{-1}+\frac{d x_{0}}{x_{0}-x_{-1}}\right) n+d n^{2}
\end{aligned}
$$

for $n \in \mathbb{N}_{0}$.

It is easy to see that Eqs. (56) and (57) match with Eq. (7), which shows that the method also leads to finding the general solution to Eq. (9) in this case.

Case $a \neq 1$. By using the change of variables (47) in Eq. (45) we obtain

$$
u_{m+1}=(a+1) u_{m}-a u_{m-1},
$$

for $m \in \mathbb{N}_{0}$.

Since the roots of the characteristic polynomial

$$
\widehat{P}_{2}(\lambda)=\lambda^{2}-(a+1) \lambda+a
$$

associated with homogeneous linear difference equation (58) are $\lambda_{1}=a, \lambda_{2}=1$, then by using the de Moivre formula (2) it follows that the general solution to Eq. (58) is

$$
u_{m}=\frac{\left(u_{0}-u_{-1}\right) a^{m+1}+a u_{-1}-u_{0}}{a-1},
$$

for $m \geq-1$.

Employing (59) in (47), we obtain

$$
\begin{aligned}
z_{m} & =\frac{\left(u_{0}-u_{-1}\right) a^{m+1}+a u_{-1}-u_{0}}{\left(u_{0}-u_{-1}\right) a^{m}+a u_{-1}-u_{0}} \\
& =\frac{\left(z_{0}-1\right) a^{m+1}+a-z_{0}}{\left(z_{0}-1\right) a^{m}+a-z_{0}},
\end{aligned}
$$

for $m \in \mathbb{N}_{0}$.

From (60), it follows that

$$
y_{2 m+i}=y_{m}^{(i)}=\frac{\left(y_{0}^{(i)}-1\right) a^{m+1}+a-y_{0}^{(i)}}{\left(y_{0}^{(i)}-1\right) a^{m}+a-y_{0}^{(i)}}=\frac{\left(y_{i}-1\right) a^{m+1}+a-y_{i}}{\left(y_{i}-1\right) a^{m}+a-y_{i}},
$$


for $m \in \mathbb{N}_{0}$ and $i=0,1$, from which together with (42) we have

$$
\begin{aligned}
y_{2 m}= & \frac{\left(y_{0}-1\right) a^{m+1}+a-y_{0}}{\left(y_{0}-1\right) a^{m}+a-y_{0}}=\frac{\left(x_{0}-x_{-1}\right) a^{m+1}+a x_{-1}-x_{0}}{\left(x_{0}-x_{-1}\right) a^{m}+a x_{-1}-x_{0}}, \\
y_{2 m+1} & =\frac{\left(y_{1}-1\right) a^{m+1}+a-y_{1}}{\left(y_{1}-1\right) a^{m}+a-y_{1}}=\frac{\left(x_{1}-x_{0}\right) a^{m+1}+a x_{0}-x_{1}}{\left(x_{1}-x_{0}\right) a^{m}+a x_{0}-x_{1}} \\
& =\frac{\left((a-1) x_{0}+\frac{d x_{0}}{x_{0} a x_{-1}}\right) a^{m+1}-\frac{d x_{0}}{x_{0} a x_{-1}}}{\left((a-1) x_{0}+\frac{d x_{0}}{x_{0}-a x_{-1}}\right) a^{m}-\frac{d x_{0}}{x_{0}-a x_{-1}}} \\
& =\frac{\left((a-1)\left(x_{0}-a x_{-1}\right)+d\right) a^{m+1}-d}{\left((a-1)\left(x_{0}-a x_{-1}\right)+d\right) a^{m}-d},
\end{aligned}
$$

for $m \in \mathbb{N}_{0}$.

By using (54), (61) and (62), we have

$$
\begin{aligned}
x_{2 n-1} & =x_{-1} \prod_{i=0}^{n-1} y_{2 i} \prod_{i=0}^{n-1} y_{2 i+1} \\
& =x_{-1} \prod_{i=0}^{n-1} \frac{\left(x_{0}-x_{-1}\right) a^{i+1}+a x_{-1}-x_{0}}{\left(x_{0}-x_{-1}\right) a^{i}+a x_{-1}-x_{0}} \prod_{i=0}^{n-1} \frac{\left((a-1)\left(x_{0}-a x_{-1}\right)+d\right) a^{i+1}-d}{\left((a-1)\left(x_{0}-a x_{-1}\right)+d\right) a^{i}-d} \\
& =\frac{\left(\left(x_{0}-x_{-1}\right) a^{n}+a x_{-1}-x_{0}\right)\left(\left((a-1)\left(x_{0}-a x_{-1}\right)+d\right) a^{n}-d\right)}{(a-1)^{2}\left(x_{0}-a x_{-1}\right)}
\end{aligned}
$$

for $n \in \mathbb{N}_{0}$

Further, from (54), (61) and (63), we get

$$
\begin{aligned}
x_{2 n}= & x_{2 n-1} y_{2 n} \\
= & \frac{\left(\left(x_{0}-x_{-1}\right) a^{n}+a x_{-1}-x_{0}\right)\left(\left((a-1)\left(x_{0}-a x_{-1}\right)+d\right) a^{n}-d\right)}{(a-1)^{2}\left(x_{0}-a x_{-1}\right)} \\
& \times \frac{\left(x_{0}-x_{-1}\right) a^{n+1}+a x_{-1}-x_{0}}{\left(x_{0}-x_{-1}\right) a^{n}+a x_{-1}-x_{0}} \\
= & \frac{\left.\left((a-1)\left(x_{0}-a x_{-1}\right)+d\right) a^{n}-d\right)\left(\left(x_{0}-x_{-1}\right) a^{n+1}+a x_{-1}-x_{0}\right)}{(a-1)^{2}\left(x_{0}-a x_{-1}\right)},
\end{aligned}
$$

for $n \in \mathbb{N}_{0}$.

It is easy to see that Eqs. (63) and (64) match with Eq. (24) (see also (29) and (31)), which shows that the method of substitution also leads to finding a general solution to Eq. (9) in this case.

Remark 1 Recall that solvability of Eq. (45) obviously implies solvability of Eq. (40). But it is not immediately clear why Eqs. (63) and (64) present a general solution to Eq. (9). To be on the safe side the formulas should be checked by induction. Nevertheless, the question needs an answer. The catch is that we consider here only well-defined solutions, that is, the solutions which satisfy condition (10). This means that for such solutions Eq. (40) is equivalent to the following one:

$$
\frac{\left(x_{n+1}-a x_{n}\right)\left(x_{n}-a x_{n-1}\right)}{d x_{n}}=\frac{\left(x_{n}-a x_{n-1}\right)\left(x_{n-1}-a x_{n-2}\right)}{d x_{n-1}}, \quad n \in \mathbb{N},
$$


which is clearly equivalent to the following one:

$$
\frac{\left(x_{n+1}-a x_{n}\right)\left(x_{n}-a x_{n-1}\right)}{d x_{n}}=\frac{\left(x_{1}-a x_{0}\right)\left(x_{0}-a x_{-1}\right)}{d x_{0}}, \quad n \in \mathbb{N} \text {. }
$$

Now note that, from (9) with $n=0$, we get

$$
\frac{\left(x_{1}-a x_{0}\right)\left(x_{0}-a x_{-1}\right)}{d x_{0}}=1
$$

From (65) and (66), we get relation (9).

Hence, a well-defined solution to Eq. (9) satisfy Eqs. (9) and (40) simultaneously.

\section{Acknowledgements}

The study in the paper is a part of the investigations under the projects III 41025 and III 44006 by the Serbian Ministry of Education and Science.

\section{Funding}

Not applicable.

\section{Availability of data and materials}

Not applicable.

\section{Competing interests}

The author declares that he has no competing interests.

\section{Authors' contributions}

The author has contributed solely to the writing of this paper. He read and approved the manuscript.

\section{Author details}

${ }^{1}$ Mathematical Institute of the Serbian Academy of Sciences, Beograd, Serbia. ${ }^{2}$ Department of Medical Research, China Medical University Hospital, China Medical University, Taichung, Taiwan, Republic of China. ${ }^{3}$ Department of Computer Science and Information Engineering, Asia University, Taichung, Taiwan, Republic of China.

\section{Publisher's Note}

Springer Nature remains neutral with regard to jurisdictional claims in published maps and institutional affiliations.

Received: 14 May 2019 Accepted: 4 June 2019 Published online: 13 June 2019

\section{References}

1. de Moivre, A.: Miscellanea analytica de seriebus et quadraturis. J. Tonson \& J. Watts, Londini (1730) (in Latin)

2. de Moivre, A.: The Doctrine of Chances, 3rd edn. London (1756)

3. Eulero, L.: Introductio in Analysin Infinitorum, Tomus Primus (1748) Lausannae (in Latin)

4. Lagrange, J.-L.: Sur l'intégration d'une équation différentielle à différences finies, qui contient la théorie des suites récurrentes, Miscellanea Taurinensia. t. I, (1759), 33-42 (Lagrange OEuvres, I, 23-36, 1867) (in French)

5. Lagrange, J.-L.: OEuvres, t. II. Gauthier-Villars, Paris (1868) (in French)

6. Laplace, P.S.: Recherches sur l'intégration des équations différentielles aux différences finies et sur leur usage dans la théorie des hasards, Mémoires de l'Académie Royale des Sciences de Paris 1773, t. VII, (1776) (Laplace OEuvres, VIII, 69-197, 1891). (in French)

7. Stević, S.: Representations of solutions to linear and bilinear difference equations and systems of bilinear difference equations. Adv. Differ. Equ. 2018, Article ID 474 (2018)

8. Boole, G.: A Treatise on the Calculus of Finite Differences, 3rd edn. Macmillan \& Co., London (1880)

9. Fort, T.: Finite Differences and Difference Equations in the Real Domain. Oxford University Press, London (1948)

10. Jordan, C.: Calculus of Finite Differences. Chelsea, New York (1956)

11. Krechmar, V.A.: A Problem Book in Algebra. Mir, Moscow (1974)

12. Levy, H., Lessman, F.: Finite Difference Equations. Dover, New York (1992)

13. Mitrinović, D.S., Adamović, D.D.: Sequences and Series. Naučna Knjiga, Beograd (1980) (in Serbian)

14. Mitrinović, D.S., Kečkić, J.D.: Methods for Calculating Finite Sums. Naučna Knjiga, Beograd (1984) (in Serbian)

15. Adamović, D.: Solution to problem 194. Mat. Vesn. 23, 236-242 (1971)

16. Stević, S.: Representation of solutions of bilinear difference equations in terms of generalized Fibonacci sequences. Electron. J. Qual. Theory Differ. Equ. 2014, Article ID 67 (2014)

17. Stević, S., Iričanin, B., Kosmala, W., Šmarda, Z.: Note on the bilinear difference equation with a delay. Math. Methods Appl. Sci. 41, 9349-9360 (2018)

18. Stević, S., Iričanin, B., Kosmala, W., Šmarda, Z.: Representation of solutions of a solvable nonlinear difference equation of second order. Electron. J. Qual. Theory Differ. Equ. 2018, Article ID 95 (2018) 
19. Stević, S., Iričanin, B., Šmarda, Z:: On a symmetric bilinear system of difference equations. Appl. Math. Lett. 89, 15-21 (2019)

20. Stević, S., Diblik, J., Iričanin, B., Šmarda, Z.: On some solvable difference equations and systems of difference equations. Abstr. Appl. Anal. 2012, Article ID 541761, 11 pp. (2012)

21. Stević, S., Diblik, J., Iričanin, B., Šmarda, Z:: On a solvable system of rational difference equations. J. Differ. Equ. Appl. 20(5-6), 811-825 (2014)

22. Stević, S., Diblik, J., Iričanin, B., Šmarda, Z.: Solvability of nonlinear difference equations of fourth order. Electron. J. Differ. Equ. 2014, Article ID 264 (2014)

23. Papaschinopoulos, G., Stefanidou, G.: Asymptotic behavior of the solutions of a class of rational difference equations. Int. J. Difference Equ. 5(2), 233-249 (2010)

24. Berg, L., Stević, S.: On some systems of difference equations. Appl. Math. Comput. 218, 1713-1718 (2011)

25. Stević, S.: On the difference equation $x_{n}=x_{n-2} /\left(b_{n}+c_{n} x_{n-1} x_{n-2}\right)$. Appl. Math. Comput. 218, 4507-4513 (2011)

26. Stević, S.: On the difference equation $x_{n}=x_{n-k} /\left(b+c x_{n-1} \cdots x_{n-k}\right)$. Appl. Math. Comput. 218, 6291-6296 (2012)

27. Stević, S.: On the system of difference equations $x_{n}=c_{n} y_{n-3} /\left(a_{n}+b_{n} y_{n-1} x_{n-2} y_{n-3}\right), y_{n}=\gamma_{n} x_{n-3} /\left(\alpha_{n}+\beta_{n} x_{n-1} y_{n-2} x_{n-3}\right)$. Appl. Math. Comput. 219, 4755-4764 (2013)

28. Stević, S.: Solvable subclasses of a class of nonlinear second-order difference equations. Adv. Nonlinear Anal. 5(2), 147-165 (2016)

29. Stević, S., Diblik, J., Iričanin, B., Šmarda, Z.: On a third-order system of difference equations with variable coefficients. Abstr. Appl. Anal. 2012, Article ID 508523, 22 pp (2012)

30. Berezansky, L., Braverman, E.: On impulsive Beverton-Holt difference equations and their applications. J. Differ. Equ. Appl. 10(9), 851-868 (2004)

31. Mitrinović, D.S.: Matrices and Determinants. Naučna Knjiga, Beograd (1989) (in Serbian)

32. Proskuryakov, I.V.: Problems in Linear Algebra. Nauka, Moscow (1984) (in Russian)

33. Riordan, J.: Combinatorial Identities. Wiley, New York (1968)

34. Stević, S.: Bounded and periodic solutions to the linear first-order difference equation on the integer domain. Adv. Differ. Equ. 2017, Article ID 283 (2017)

35. Stević, S.: Bounded solutions to nonhomogeneous linear second-order difference equations. Symmetry 9, Article ID $227(2017)$

36. Stević, S.: Existence of a unique bounded solution to a linear second order difference equation and the linear first order difference equation. Adv. Differ. Equ. 2017, Article ID 169 (2017)

37. Alfred, B.U.: An Introduction to Fibonacci Discovery. The Fibonacci Association (1965)

38. Andruch-Sobilo, A., Migda, M.: Further properties of the rational recursive sequence $x_{n+1}=a x_{n-1} /\left(b+c x_{n} x_{n-1}\right)$. Opusc. Math. 26(3), 387-394 (2006)

39. Eves, H., Starke, E.P., eds., The Otto Dunkel Memorial Problem Book, Amer. Math. Monthly, 64 (1957)

40. Iričanin, B., Stević, S.: Eventually constant solutions of a rational difference equation. Appl. Math. Comput. 215 854-856 (2009)

41. Stević, S.: On some periodic systems of max-type difference equations. Appl. Math. Comput. 218, 11483-11487 (2012)

42. Vorobiev, N.N.: Fibonacci Numbers. Birkhäuser, Basel (2002) (Russian original 1950)

43. Elsayed, E.M.: Dynamics of recursive sequence of order two. Kyungpook Math. J. 50(4), 483-497 (2010)

44. Stević, S.: Solvability of a one-parameter class of nonlinear second-order difference equations by invariants. Adv. Differ. Equ. 2019, Article ID 151 (2019)

45. Papaschinopoulos, G., Schinas, C.J.: On the behavior of the solutions of a system of two nonlinear difference equations. Commun. Appl. Nonlinear Anal. 5(2), 47-59 (1998)

46. Papaschinopoulos, G., Schinas, C.J.: Invariants for systems of two nonlinear difference equations. Differ. Equ. Dyn. Syst. 7, 181-196 (1999)

47. Papaschinopoulos, G., Schinas, C.J.: Invariants and oscillation for systems of two nonlinear difference equations. Nonlinear Anal., Theory Methods Appl. 46, 967-978 (2001)

48. Papaschinopoulos, G., Schinas, C.J., Stefanidou, G.: On a k-order system of Lyness-type difference equations. Adv. Differ. Equ. 2007, Article ID 31272 (2007)

49. Schinas, C.: Invariants for difference equations and systems of difference equations of rational form. J. Math. Anal. Appl. 216, 164-179 (1997)

50. Schinas, C.: Invariants for some difference equations. J. Math. Anal. Appl. 212, 281-291 (1997)

51. Stević, S.: Solvability of some classes of nonlinear first-order difference equations by invariants and generalized invariants. Electron. J. Qual. Theory Differ. Equ. 2019, Article ID 36 (2019)

52. Stević, S.: Solving a class of non-autonomous difference equations by generalized invariants. Math. Methods Appl. Sci. 42 (2019, in press)

53. Stević, S.: First-order product-type systems of difference equations solvable in closed form. Electron. J. Differ. Equ. 2015, Article ID 308 (2015)

54. Stević, S.: Product-type system of difference equations of second-order solvable in closed form. Electron. J. Qual. Theory Differ. Equ. 2015, Article ID 56 (2015)

55. Stević, S.: Solvable product-type system of difference equations whose associated polynomial is of the fourth order. Electron. J. Qual. Theory Differ. Equ. 2017, Article ID 13 (2017) 